\title{
Geodesic Structure of Lifshitz Black Holes in 2+1 Dimensions
}

\author{
Norman Cruz* \\ Departamento de Física, Facultad de Ciencia, \\ Universidad de Santiago de Chile, Casilla 307, \\ Santiago, Chile \\ Marco Olivares $\dagger$ \\ Instituto de Física, \\ Pontificia Universidad de Católica de Valparaíso, \\ Av. Universidad 330, Curauma, \\ Valparaíso, Chile \\ J. R. Villanueva \\ Departamento de Física y Astronomía, Facultad de Ciencias, \\ Universidad de Valparaíso, Gran Bretaña 1111, \\ Valparaiso, Chile, and \\ Centro de Astrofísica de Valparaíso, \\ Gran Bretaña 1111, Playa Ancha, \\ Valparaíso, Chile. \\ (Dated: July 11, 2018)
}

We present a study of the geodesic equations of a black hole space-time which is a solution of the three-dimensional NMG theory and is asymptotically Lifshitz with $z=3$ and $d=1$ as found in [Ayon-Beato E., Garbarz A., Giribet G. and Hassaine M., Phys. Rev. D 80, 104029 (2009)]. By means of the corresponding effective potentials for massive particles and photons we find the allowed motions by the energy levels. Exact solutions for radial and non-radial geodesics are given in terms of the Weierstrass elliptic $\wp, \sigma$, and $\zeta$ functions.

PACS numbers: 04.20.Fy, 04.20.Jb, 04.40.Nr, 04.70.Bw

Keywords: Black Holes; Elliptic Functions.

\section{Contents}

\section{Introduction}

II. Geodesics of the Lifshitz black hole space-time

A. Time Like Geodesics

1. Angular Motion

2. Radial Motion

B. Null Geodesics

1. Angular Motion

2. Radial Motion

\section{Final Remarks}

Acknowledgments

References

\footnotetext{
*Electronic address: norman.cruz@usach.cl

${ }^{\dagger}$ Electronic address: marco.olivaresrubilar@gmail.com

‡Electronic address: jose.villanuevalob@uv.cl
} 


\section{INTRODUCTION}

The New Massive Gravity (NMG) [4] theory has deserved special attention due to its remarkable physical properties. Since lower-dimensional gravity has been an active field of research in order to shed some light on the behavior of classical and quantum aspects of this interaction, models in $(2+1)$ dimensions have attracted a lot of research. In the case of NMG its $(2+1)$-dimensional version is described by an action defined by

$$
S=\frac{1}{16 \pi G_{3}} \int d^{3} x \sqrt{-g}\left[R-2 \lambda-\frac{1}{m^{2}}\left(R_{\mu \nu} R^{\mu \nu}-\frac{3}{8} R^{2}\right)\right],
$$

where $G_{3}$ is the three-dimensional Newton constant, $m$ is the "relative mass parameter, and $\lambda$ is the three-dimensional cosmological constant. The minus sign in the Einstein-Hilbert term avoids negative mass and entropy. This action gives rise to field equations with a second order trace. Its linearized version is equivalent to the Fierz-Pauli action for a massive spin-2 field in three dimension, so it is a unitary model [15]. This theory admits black hole-type solutions asymptotically Lifshitz, characterized by the metrics [12]

$$
d s^{2}=-\frac{r^{2 z}}{\ell^{2 z}} d t^{2}+\frac{\ell^{2}}{r^{2}} d r^{2}+\frac{r^{2}}{\ell^{2}} d \vec{x}^{2}
$$

where $\vec{x}$ is a $d$-dimensional vector. These space-times present invariance under the rescaling $(t, \vec{x}, r) \mapsto\left(\lambda^{z} t, \lambda \vec{x}, \lambda^{-1} r\right)$. Many investigations have addressed to find black hole solutions which asymptote the metrics given by (2). A fourdimensional topological black hole which is asymptotically Lifshitz with dynamical exponent $z=2$ was found in 13 . Other Lifshitz black hole solutions were investigated in [5, 9]. A black hole solution for $z=2$ in $d=2$ was found in [2].

In this paper our aim is to investigate the geodesic structure of black hole space-time which is a solution of the three-dimensional NMG theory and is asymptotically Lifshitz with $z=3$ and $d=1$ [1]. Similar studies have been made for Topological Lifshitz black hole in 3+1 dimensions [16]. We investigate all the possible movements allowed in this space-time by means of a detailed analysis of the effective potentials. We find the exact solutions describing the path of massive particles and photons. In order to obtain a direct visualization of the allowed motions, we plot the orbits found.

In section II, we derive the geodesic equations of motion using the variational problem associated with the metric of the black hole space-time found in [1]. For both massive particles and photons the following issues are investigated: i) the effective potential of the equivalent one-dimensional problem, which allows us to restrict the motions if they are radial or presents a non-zero angular momentum, ii) the exact solution for radial geodesics in terms of the proper and coordinate time, and iii) exact solutions for the polar equation in motions with angular momentum. The exact solutions are given in terms of the Weierstrass elliptic $\wp, \sigma$, and $\zeta$ functions. In section III we summarize and discuss our results.

\section{GEODESICS OF THE LIFSHITZ BLACK HOLE SPACE-TIME}

The three-dimensional Lifshitz black hole solutions presented in Ref. [1] are a family (which generic values of the dynamical exponent $z$ ) described by the following metric:

$$
d s^{2}=-\frac{r^{2 z}}{\ell^{2 z}} F(r) d t^{2}+\frac{\ell^{2}}{r^{2}} H(r) d r^{2}+r^{2} d \phi^{2},
$$

where the coordinate are defined as $-\infty \leq t \leq \infty, r \geq 0,0 \leq \phi \leq 2 \pi$ and functions $F(r)$ and $H(r)$ depends only of the radial coordinate. Besides, these function take the value $F(r)=H(r)=1$ when $r \rightarrow \infty$, which means that the metrics are asymptotically Lifshitz. In order to have a single horizon it was required that these functions present a single-zero at location of the horizon $\left(r_{+}\right)$. For the case $z=3$ the equations of motions are solved choosing the following form for the functions $F(r)$ and $H(r)[1]$ :

$$
F(r)=H^{-1}(r)=\left(1-\frac{M \ell^{2}}{r^{2}}\right)
$$

where $M$ is an integration constant and $\ell$ is the length associated to the three-dimensional cosmological constant. This solution corresponds to the static asymptotically Lifshitz black hole, whose metric can be written as

$$
d s^{2}=-\frac{r^{4} \Delta}{\ell^{6}} d t^{2}+\frac{\ell^{2}}{\Delta} d r^{2}+r^{2} d \phi^{2}
$$


where $\Delta=r^{2}-r_{+}^{2}$. The Kretschmann scalar associated to this metric is given by

$$
R_{\mu \nu \lambda \sigma} R^{\mu \nu \lambda \sigma}=-\frac{4\left(8 r_{+}^{4}-48 r_{+}^{2} r^{2}+91 r^{4}\right)}{\ell^{4} r^{4}}
$$

which diverges for $r \rightarrow 0$. For $r \rightarrow \infty$ the scalar takes the value $-364 / \ell^{4} \sim \lambda^{2}$, which characterize an AdS space-time. The curvature invariants $R$ and $R_{\mu \nu} R^{\mu \nu}$ also diverge at $r=0$ [1] indicating a curvature singularity at the origin. A single horizon is located at $r_{+}=\ell \sqrt{M}$. The case $z=1$ described the well known BTZ black hole [3]. The geodesic motion on this space-time was first studied in [10] and a general study of its structure geodesic was explored in [7].

Let us mention some differences of the space-time described by the metric (5) and the BTZ black hole. Contrary to our intuition in $3+1$ dimensions where gravity increases as the distance to the center of force diminishes (and so does the curvature), which is a property of the Schwarzschild metric; in the BTZ black hole the curvature is constant and negative. Nevertheless, the Lifshitz black hole solution has a variable curvature as Eq.(6) shows. For the BTZ black hole $M$ is the conserved charge associated with asymptotic invariance under displacement, so it can be identified with the black hole mass. In the case of Lifshitz black hole, its mass has been evaluated in [14], using the Euclidean action approach [11] with the action given in [4]. The expression found for the mass, $\mathcal{M}$, is

$$
\mathcal{M}=\frac{r_{+}^{4}}{4 G_{3} \ell^{4}}=\frac{M^{2}}{4 G_{3}}
$$

indicating the relationship between the constant of integration which appears in the metric (55) and the black hole mass.

In order to compute the geodesic structures of the Lifshitz black hole, we start from the Lagrangian given by

$$
2 \mathfrak{L}=-\frac{r^{4} \Delta}{\ell^{6}} \dot{t}^{2}+\frac{\ell^{2}}{\Delta} \dot{r}^{2}+r^{2} \dot{\phi}^{2}=-h,
$$

where a dot corresponds to the derivative with respect to an affine parameter, $\tau$, along the trajectory, and $h=0$ for massless particles and $h=1$ for massive particles. Considering that the Lagrangian (8) is independent of $(t, \phi)$, their corresponding conjugate momenta, $\left(\Pi_{t}, \Pi_{\phi}\right)$, are conserved, and are given by

$$
\sqrt{E}=\frac{r^{4} \Delta}{\ell^{6}} \dot{t}, \quad \text { and } \quad L=r^{2} \dot{\phi}
$$

respectively. Using these constants of motion in the radial equation we can write the radial quadratures in $(\tau, t, \phi)$ as

$$
\begin{gathered}
\left(\frac{d r}{d \tau}\right)^{2}=\frac{\ell^{4}}{r^{4}}\left[E-V_{L i f}(r)\right], \\
\left(\frac{d r}{d t}\right)^{2}=\frac{r^{4} \Delta^{2}}{\ell^{8} E^{2}}\left[E-V_{L i f}(r)\right],
\end{gathered}
$$

and

$$
\left(\frac{d r}{d \phi}\right)^{2}=\frac{\ell^{4}}{L^{2}}\left[E-V_{L i f}(r)\right]
$$

where $V_{L i f}(r)$ is the so-called effective potential for the $2+1$ Lifshitz space-time, which is given by

$$
V_{L i f}(r)=\frac{r^{4} \Delta}{\ell^{6}}\left(h+\frac{L^{2}}{r^{2}}\right) .
$$

It is well known that an extreme of the effective potential, located at some point $r_{c, h}$, gives us information on the existence and stability of the circular orbits, and also information on the structure of space-time. For example, under certain circumstances the existence of a stable circular orbit would give the possibility to have confined orbits. Thus, considering (13) we obtain the following equality:

$$
L^{2}\left(2 r_{c, h}^{2}-r_{+}^{2}\right)+h r_{c, h}^{2}\left(3 r_{c, h}^{2}-2 r_{+}^{2}\right)=0
$$


which for massless particles has the solution

$$
r_{c, 0}=\frac{r_{+}}{\sqrt{2}} \equiv R_{c} \approx 0.71 r_{+} .
$$

This result is independent of the angular momentum and smaller than the event horizon. On the other hand, for massive particles we obtain

$$
r_{c, 1}=\sqrt{\frac{r_{+}^{2}+\sqrt{L^{4}+L^{2} r_{+}^{2}+r_{+}^{4}}-L^{2}}{3}},
$$

which is always smaller than the event horizon, independent of the value of the angular momentum. Also, note that

$$
\begin{aligned}
& r_{c, 1} \rightarrow R_{c}\left(1+\frac{1}{4} \frac{R_{c}^{2}}{L^{2}}+0\left(R_{c} / L\right)^{3}\right) \approx 0.71 r_{+}, \quad \text { if } \quad L \rightarrow \infty, \\
& r_{c, 1} \rightarrow \frac{2 \sqrt{3}}{3} R_{c}\left(1-\frac{1}{16} \frac{L^{2}}{R_{c}^{2}}+0\left(L / R_{c}\right)^{3}\right) \approx 0.82 r_{+}, \quad \text { if } \quad L \rightarrow 0 .
\end{aligned}
$$

Therefore, from (15) and (16) we conclude that confined orbits are forbidden in the $2+1$ Lifshitz background. This last fact has also been found recently in a topological Lifshitz black hole in $3+1$ dimensions [16].

\section{A. Time Like Geodesics}

In this case $h=1$, and the effective potential (13) can be rewritten in the following form:

$$
V_{t}(r)=\frac{r^{4}}{\ell^{6}}\left(r^{2}-r_{+}^{2}\right)\left(1+\frac{L^{2}}{r^{2}}\right) .
$$

In Fig. 1 we have plotted this potential for particles with null angular momentum $L=0$ and non-vanished angular momentum $L \neq 0$.

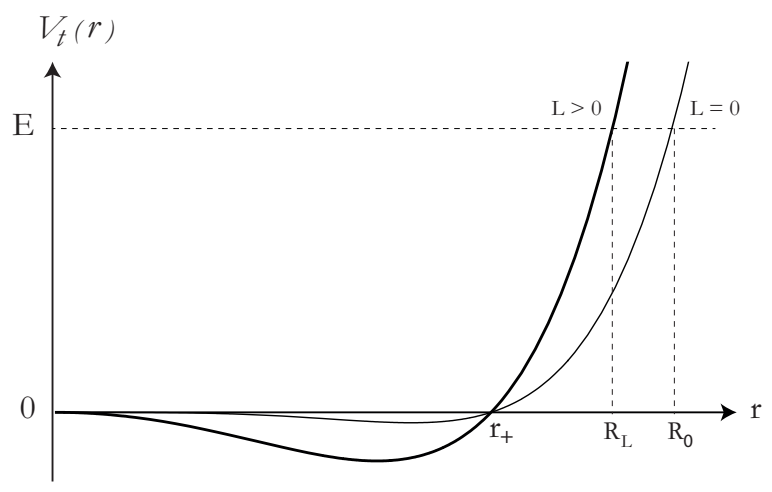

FIG. 1: Effective potential for massive particles. As the angular momentum increase, the maximum distance that the particle can reach diminishes, for a given $E$.

\section{Angular Motion}

Angular motions of massive particles are characterized by $L>0$, such that the effective potential yields

$$
V_{t a}(r)=\frac{r^{4}}{\ell^{6}}\left(r^{2}-r_{+}^{2}\right)\left(1+\frac{L^{2}}{r^{2}}\right)
$$

Thus, the turning point, $R_{L}$, is given by 


$$
R_{L}^{2}=\frac{2}{3} \sqrt{r_{+}^{4}+r_{+}^{2} L^{2}+L^{4}} \cosh \left[\frac{1}{3} \cosh ^{-1}\left(\frac{27 E \ell^{6}+2 r_{+}^{6}+3 r_{+}^{4} L^{2}-3 r_{+}^{2} L^{4}-2 L^{6}}{2 \sqrt{\left(r_{+}^{4}+r_{+}^{2} L^{2}+L^{4}\right)^{3}}}\right)\right]+\frac{r_{+}^{2}-L^{2}}{3}
$$

and the quadrature (10) can be written as

$$
\phi(r)=L \ell \int_{R_{L}}^{r} \frac{-d r}{\sqrt{E \ell^{6}+r_{+}^{2} L^{2} r^{2}+\left(r_{+}^{2}-L^{2}\right) r^{4}-r^{6}}} .
$$

Therefore, after an integration we obtain the polar trajectory,

$$
r(\phi)=\frac{\ell}{\sqrt{4 \wp\left(k \phi+\varpi ; g_{2}, g_{3}\right)-\delta_{0}}},
$$

where $\wp\left(y ; g_{2}, g_{3}\right)$ is the P-Weierstrass function, $g_{2}$ and $g_{3}$ are the so-called Weierstrass invariants given by

$$
g_{2}=\frac{r_{+}^{4} L^{4}}{12 E^{2} \ell^{8}}-\frac{r_{+}^{2}-L^{2}}{4 E \ell^{2}}, \quad \text { and } \quad g_{3}=\frac{1}{16 E}+\frac{r_{+}^{2} L^{2}\left(r_{+}^{2}-L^{2}\right)}{48 E^{2} \ell^{6}}-\frac{r_{+}^{6} L^{6}}{216 E^{3} \ell^{12}},
$$

where the constants are

$$
\delta_{0}=\frac{r_{+}^{2} L^{2}}{3 E \ell^{4}}, \quad k=\frac{2 \ell \sqrt{E}}{L}, \quad \text { and } \quad \varpi=\left|\wp^{-1}\left(\frac{1}{4}\left(\delta_{0}+\frac{\ell^{2}}{R_{L}^{2}}\right) ; g_{2}, g_{3}\right)\right| .
$$

The polar trajectory (23) is plotted in Fig. 2

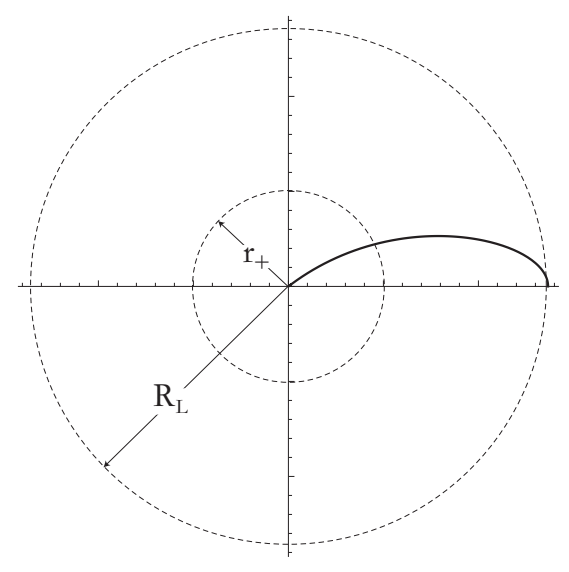

FIG. 2: Polar plot of the trajectories for massive particles with non-vanished angular momentum $L>0$.

\section{Radial Motion}

In this cases we find that $L=0$ and the effective potential (19) becomes

$$
V_{t r}(r)=\frac{r^{4}}{\ell^{6}}\left(r^{2}-r_{+}^{2}\right)
$$

whose behaviors is showed in Fig. 1, and the quadrature (10) can be written as

$$
\tau(r)=\ell \int_{R_{0}}^{r} \frac{-r^{2} d r}{\left.\sqrt{\left(R_{0}^{2}-r^{2}\right)\left[r^{4}+\left(R_{0}^{2}-r_{+}^{2}\right) r^{2}+R_{0}^{2}\left(R_{0}^{2}-r_{+}^{2}\right)\right.}\right]} .
$$


The turning point, $R_{0}$, is given by

$$
R_{0}^{2}=\frac{r_{+}^{2}}{3}+\frac{2 r_{+}^{2}}{3} \cosh \left[\frac{1}{3} \cosh ^{-1}\left(1+\frac{27 E \ell^{6}}{2 r_{+}^{6}}\right)\right] .
$$

So, after an integration of Eq. (27) we obtain

$$
\tau(r)=\frac{\ell R_{0}}{2 \sqrt{3 R_{0}^{2}-2 r_{+}^{2}}}\left[\left(1+\frac{\zeta(\Omega)}{2 \wp^{\prime}(\Omega)}\right) \wp^{-1}(U)+\frac{1}{4 \wp^{\prime}(\Omega)} \ln \left(\frac{\sigma\left(\wp^{-1}(U)-\Omega\right)}{\sigma\left(\wp^{-1}(U)+\Omega\right)}\right)\right],
$$

where $\wp^{-1}(Y) \equiv \wp^{-1}\left(Y ; g_{2}, g_{3}\right)$ is the inverse P-Weierstrass function, $\sigma(Y) \equiv \sigma\left(Y ; g_{2}, g_{3}\right)$ is the sigma Weierstrass function, $\zeta(Y) \equiv \zeta\left(Y ; g_{2}, g_{3}\right)$ is the zeta Weierstrass function, and the Weierstrass invariants are given by

$$
g_{2}=\frac{1}{12}\left(A^{2}-A+1-3 B\right), \quad g_{3}=\frac{1}{432}(A-2)\left(2 A^{2}+A-1-9 B\right),
$$

with

$$
A=\frac{2 R_{0}^{2}-r_{+}^{2}}{3 R_{0}^{2}-2 r_{+}^{2}}, \quad \text { and } \quad B=\frac{R_{0}^{2}}{3 R_{0}^{2}-2 r_{+}^{2}} .
$$

Also, the radial function $U=U(r)$ and the constant $\Omega$ are given by

$$
U(r)=\frac{1}{4}\left[\frac{R_{0}^{2}}{R_{0}^{2}-r^{2}}-\frac{2 R_{0}^{2}-r_{+}^{2}}{3 R_{0}^{2}-2 r_{+}^{2}}\right], \quad \text { and } \quad \Omega=\wp^{-1}\left(\frac{1+A}{12}\right) .
$$

On the other side, from Eq.(11) we obtain the quadrature

$$
t(r)=\ell^{7} \sqrt{E} \int_{R_{0}}^{r} \frac{-d r}{r^{2}\left(r^{2}-r_{+}^{2}\right) \sqrt{E \ell^{6}+r_{+}^{2} r^{4}-r^{6}}} .
$$

Thus, making the change of variable $x=\ell^{2} / r^{2}$, and then integrating the remaining expression, we obtain

$t(r)=\frac{\ell^{5}}{r_{+}^{4}}\left\{\frac{\wp(w)}{\wp^{\prime}(w)}\left[2\left(\alpha-\wp^{-1}\left(\frac{\ell^{2}}{r^{2}}\right)\right) \zeta(w)+\beta-\ln \left(\frac{\sigma\left(\wp^{-1}\left(\frac{\ell^{2}}{r^{2}}\right)-w\right)}{\sigma\left(\wp^{-1}\left(\frac{\ell^{2}}{r^{2}}\right)+w\right)}\right)\right]+\alpha-\wp^{-1}\left(\frac{\ell^{2}}{r^{2}}\right)+\frac{\zeta\left[\wp^{-1}\left(\frac{\ell^{2}}{r^{2}}\right)\right]-\zeta(\alpha)}{\wp(w)}\right\}$.

In this case the Weierstrass invariants are given by

$$
g_{2}=-\frac{4 r_{+}^{2}}{E \ell^{2}}, \quad \text { and } \quad g_{3}=\frac{4}{E},
$$

together with the constants

$$
w=\wp^{-1}\left(\frac{\ell^{2}}{r_{+}^{2}}\right), \quad \alpha=\wp^{-1}\left(\frac{\ell^{2}}{R_{0}^{2}}\right), \quad \text { and } \quad \beta=\ln \left(\frac{\sigma(\alpha-w)}{\sigma(\alpha+w)}\right) .
$$

In Fig. 3 we plot the proper time, Eq. (29), and the coordinate time, Eq. (34), as a function of the radial coordinate, $r$. We can see that radial massive particles cross the event horizon in a finite proper time. However, an external observer will see that the particle falls asymptotically toward the event horizon. This result is common with the $3+1$ Einstein space-times (Schwarzschild [6], SAdS [8], etc.)

\section{B. Null Geodesics}

The motion of massless particles is described by the effective potential (13) with $h=0$,

$$
V_{n}(r)=\frac{L^{2}}{\ell^{6}}\left(r^{2}-r_{+}^{2}\right) r^{2},
$$

which we draw in Fig. 4. As we have shown, confined orbits are forbidden in this space-time, even though we can determine explicitly the trajectories of massless particles. 


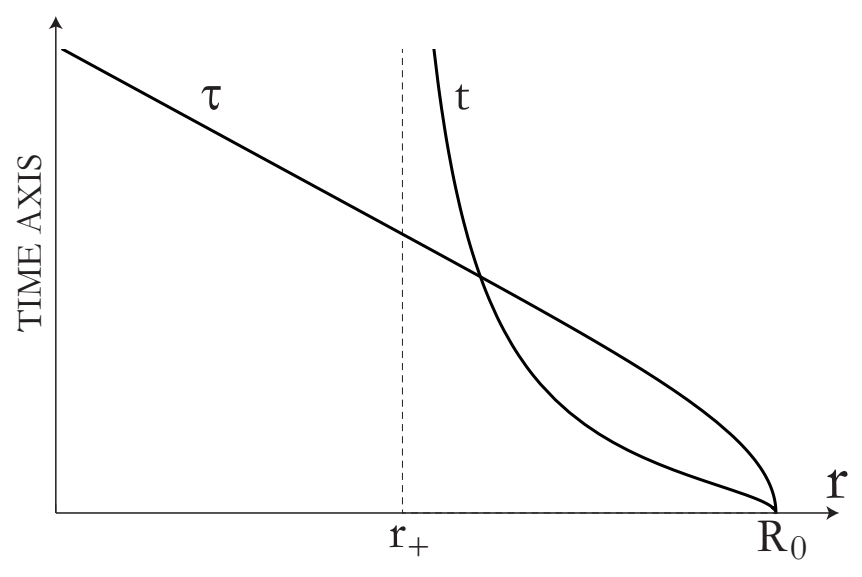

FIG. 3: Radial massive particles in the proper and coordinate time.

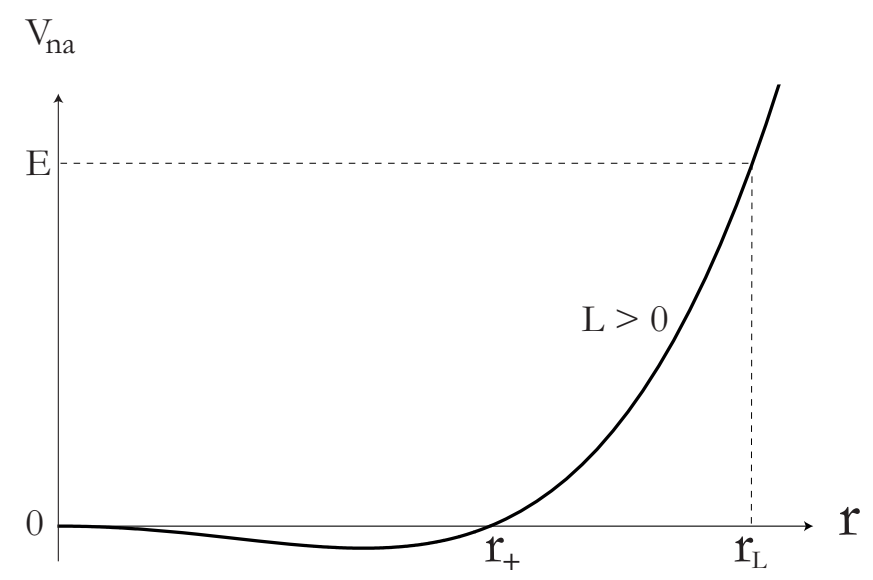

FIG. 4: Effective potential for massless particles with non-vanished angular momentum $L>0$.

\section{Angular Motion}

From Eq. (12), with $L>0$ and defining the impact parameter as $b=L / E$, it is possible to write the angular quadrature as

$$
\phi(r)=-\int_{r_{L}}^{r} \frac{d r}{\sqrt{\frac{\ell^{4}}{b^{2}}-\frac{r^{2}}{\ell^{2}}\left(r^{2}-r_{+}^{2}\right)}}=-\ell \int_{r_{L}}^{r} \frac{d r}{\sqrt{\left(r_{L}^{2}-r^{2}\right)\left(\rho_{L}^{2}+r^{2}\right)}},
$$

where $r_{L}$ is the turning point, and $\rho_{L}$ is a negative root without physical meaning, which are given by

$$
r_{L}=r_{+}\left[\frac{1}{2}+\sqrt{\frac{1}{4}+\frac{\ell^{6}}{r_{+}^{4} b^{2}}}\right]^{1 / 2} \quad \rho_{L}=r_{+}\left[-\frac{1}{2}+\sqrt{\frac{1}{4}+\frac{\ell^{6}}{r_{+}^{4} b^{2}}}\right]^{1 / 2},
$$

respectively. Thus, integrating Eq. (38) the polar form of the trajectory is obtained in terms of the P-Weierstrass function

$$
r(\phi)=r_{L}-\frac{\ell}{4 \wp\left(\kappa \phi ; g_{2}, g_{3}\right)+\delta},
$$

where the Weierstrass invariants are given in this case by

$$
g_{2}=-\frac{\ell^{2}\left(14 r_{L}^{2} \rho_{L}^{2}-r_{L}^{4}-\rho_{L}^{4}\right)}{48 r_{L}^{2}\left(r_{L}^{2}+\rho_{L}^{2}\right)^{2}}, \quad g_{3}=-\frac{\ell^{3}\left(r_{L}^{2}-\rho_{L}^{2}\right)\left(r_{L}^{4}+34 r_{L}^{2} \rho_{L}^{2}+\rho_{L}^{4}\right)}{1728 r_{L}^{3}\left(r_{L}^{2}+\rho_{L}^{2}\right)^{3}}
$$


where the constants are

$$
\kappa=\sqrt{\frac{2 r_{L}\left(r_{L}^{2}+\rho_{L}^{2}\right)}{\ell^{3}}} \quad \delta=\frac{\ell}{6 r_{L}}+\frac{2 \ell r_{L}}{3\left(r_{L}^{2}+\rho_{L}^{2}\right)} .
$$

In Fig 5 we plot the photons trajectory given by expression (40).

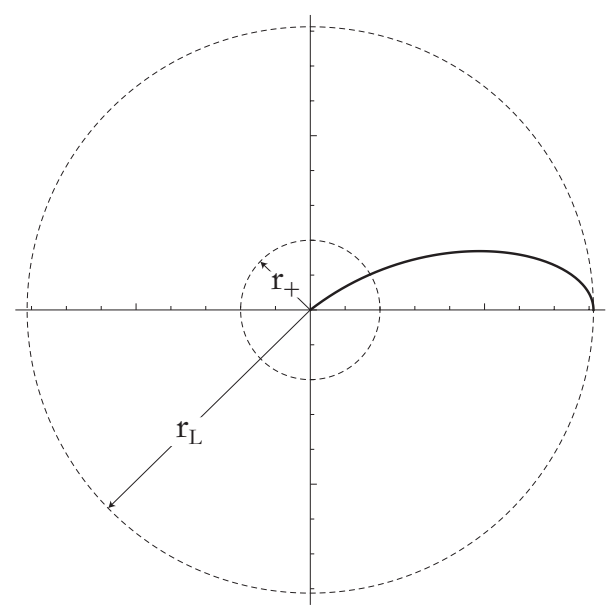

FIG. 5: Polar plot for massless particles with $L>0$.

\section{Radial Motion}

In the case of the radial motion of photons $(L=0)$, the effective potential (37) becomes

$$
V_{n r}=0,
$$

and therefore, radial photons can escape to infinity. The dynamic equations are given by

$$
\pm \frac{d r}{d \tau}=\frac{\ell^{2} \sqrt{E}}{r^{2}}
$$

and

$$
\pm \frac{d r}{d t}=\frac{r^{2} \Delta}{\ell^{4}}
$$

Integrating Eq.(44) we obtain

$$
\tau(r)= \pm \frac{1}{3 \ell^{2} \sqrt{E}}\left(r^{3}-r_{0}^{3}\right) .
$$

The minus sign represents photons that are falling toward the black hole, taking a finite proper time, $\tau_{+} \equiv \tau\left(r_{+}\right)$, to cross the event horizon; the plus sign means that the photons escape to spatial infinity, taking an infinite proper time (see Fig. 6).

On the other hand, the quadrature (45) can be written as

$$
t(r)= \pm \ell^{4} \int_{r_{0}}^{r} \frac{d r}{r^{2}\left(r^{2}-r_{+}^{2}\right)},
$$

so, after a brief manipulation in partial fractions, and then integrating, we find that the solution is

$$
t(r)= \pm \ell^{4}\left[\frac{1}{2 r_{+}^{3}} \ln \left|\frac{\left(r-r_{+}\right)\left(r_{0}+r_{+}\right)}{\left(r+r_{+}\right)\left(r_{0}-r_{+}\right)}\right|+\frac{1}{r_{+}^{2}}\left(\frac{1}{r}-\frac{1}{r_{0}}\right)\right] .
$$


This last expression shows that photons traveling to $r_{+}$have a similar behavior as the static spherically symmetric space-times of Einstein's theory, i. e., an external system will see that photons fall asymptotically to $r_{+}$. Moreover, as we have mentioned above, photons take an infinite proper time traveling to infinity, but an external system will see that photons arrives at infinite in a finite coordinate time, $t_{1}$, which is given by

$$
t_{1}=\lim _{r \rightarrow \infty} t(r)=\ell^{4}\left[\frac{1}{2 r_{+}^{3}} \ln \left(\frac{r_{0}+r_{+}}{r_{0}-r_{+}}\right)-\frac{1}{r_{+}^{2} r_{0}}\right] .
$$

This behavior is shown in Fig. [6, and has been reported recently in the topological Lifshitz 3+1 counterpart [16].

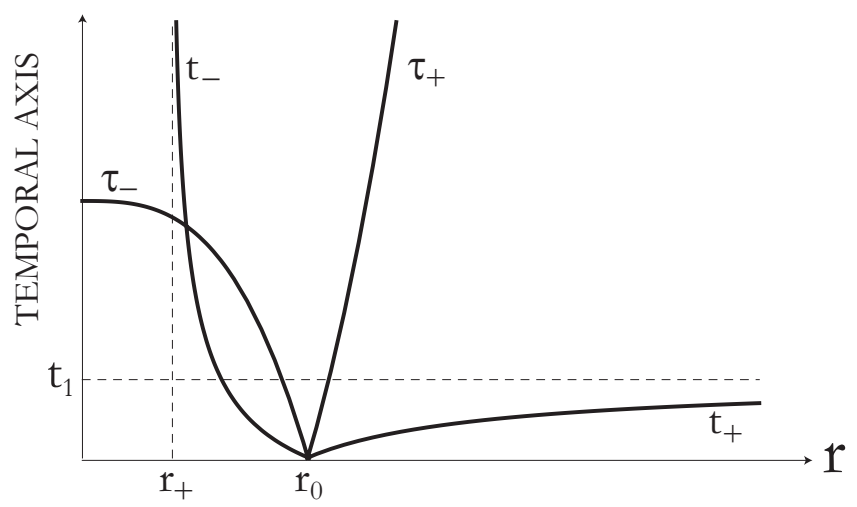

FIG. 6: Radial massless particles in the proper and coordinate time.

\section{FINAL REMARKS}

In this work we have studied the geodesic structure of the Lifshitz black hole in $2+1$ dimensions by means of an analysis of the motion of massive and massless particles in this background. Using the standard Lagrangian procedure we obtain the equations of motion in terms of the energy, $E$, and angular momentum, $L$, which as a result are found to be constants of motion for the particles. Also, we obtain an effective potential, $V(r)$, that allows us to describe qualitatively the movement, and then, we obtain an analytical solution to equations of motion.

In the first place, we found that confined orbits are forbidden (except for radial motion of massless particles), because the minimum of the effective potential as a result is found to be smaller than the event horizon, $r_{+}$.

For massive particles we found that the radial motion is similar to the Schwarzschild case: in the proper system, they cross the horizon in a finite time, whereas an external system will observe that they take an infinite time to do it. We also found the trajectories of massive particles falling into the horizon with $L>0$ in terms of the elliptic $\wp$-Weierstrass function.

On the other side, the motion of massless particles with $L>0$ has the same general behavior of massive particles. Moreover, photons with vanishing angular momentum can escape to spatial infinity: in the proper system they take an infinity of time to escape, but an external system will observe that they take a finite time, $t_{1}$, to do it, as shown in Eq. (49). This last fact also has been found in the Topological Lifshitz black hole [16].

\section{Acknowledgments}

M. O. thanks to PUCV. This work was supported by DICYT-USACH Grant No 041331CM (NC).

[1] Ayon-Beato E., Garbarz A., Giribet G. and Hassaine M.: Lifshitz Black Hole in Three Dimensions. Phys. Rev. D 80, 104029 (2009) [arXiv: 0909.1347].

[2] Balasubramanian K. and McGreevy J.: An analytic Lifshitz black hole. Phys. Rev. D 80, 104039 (2009) [arXiv: 0909.0263]. 
[3] Bañados M., Teitelboim C. and Zanelli J.: The Black Hole in three-dimensional Space Time. Phys. Rev. Lett. 69, 1849 (1992) [arXiv: 9204099].; Bañados M., Henneaux M., Teitelboim C. and Zanelli J.: Geometry of the 2+1 Black Hole. Phys. Rev. D 48, 1506 (1993) [arXiv: 9302012].

[4] Bergshoeff E., Hohm O. and Townsend P.: Massive Gravity in Three Dimensions. Phys. Rev. Lett. 102 201301 (2009) [arXiv: 0901.1766].

[5] Brynjolfsson E., Danielsson U., Thorlacius L. and Zingg T.: Holographic Superconductors with Lifshitz Scaling. J. Phys. A 43, 065401 (2010) [arXiv: 0908.2611].

[6] Chandrasekhar S.: The Mathematical Theory of Black Holes. Oxford University Press, New York (1983).

[7] Cruz N., Martínez C. and Peña L.: Geodesic structure of the $(2+1)$-dimensional BTZ black hole. Class. Quantum Grav. 11, 2731 (1994) [arXiv: 9401025].

[8] Cruz N., Olivares M. and Villanueva J. R.: The geodesic structure of the Schwarzschild anti-de Sitter Black Hole. Class. Quantum Grav. 22, 1167-1190 (2005) [arXiv: 0408016].

[9] Danielsson U. and Thorlacius L.: Black holes in asymptotically Lifshitz space-time. JHEP 0903, 070 (2009) [arXiv: 0812.5088].

[10] Farina C., Gamboa J. and Seguí-Santonja A. J.: Motion and Trajectories of Particles Around Three-Dimensional Black Holes. Class. Quantum Grav. 10, 193 (1993) [arXiv: 9303005].

[11] Gonzalez H. A., Tempo D. and Troncoso R.: Field theories with anisotropic scaling in 2D, solitons and the microscopic entropy of asymptotically Lifshitz black holes. JHEP, 1111, 066 (2011) [arXiv: 1107.3647].

[12] Kachru S., Liu X. and Mulligan M.: Gravity Duals of Lifshitz-like Fixed Points. Phys. Rev. D 78, 106005 (2008) [arXiv: 0808.1725].

[13] Mann R. B.: Lifshitz topological black holes. JHEP 06, 075 (2009). [arXiv: 0905.1136].

[14] Myung Y. S.: Phase transitions for the Lifshitz black holes. Eur. Phys. J. C 722116 (2012) [arXiv: 1203.1367].

[15] Nakasone M. and Oda I.: On Unitarity of Massive Gravity in Three Dimensions. Prog. Theor. Phys. 121, 1389 (2009) [arXiv: 0902.3531]; Deser S.: Ghost-free, finite, fourth order D=3 (alas) gravity. Phys. Rev. Lett. 103, 101302 (2009) [arXiv: 0904.4473].

[16] Olivares M., Rojas G., Vásquez Y. and Villanueva J. R.: Particles motion on topological Lifshitz black holes in 3+1 dimensions. Accepted for publication in Astrophys. Space Sci. (2013) [arXiv: 1304.4297]. 